\title{
Design of Natural Food Antioxidant Ingredients through a Chemometric Approach
}

\author{
Jose A. Mendiola ${ }^{\dagger}$ Pedro J. Martín-Álvarez, ${ }^{\dagger}$ F. Javier Señoráns, ${ }^{\$}$ \\ Guillermo Reglero, "Alessandro Capodicasa, ${ }^{\S}$ Filomena Nazzaro, \\ Alfonso Sada," Alejandro Cifuentes, ${ }^{\dagger}$ And Elena Ibáñez ${ }^{*}, \dagger$ \\ ${ }^{\dagger}$ Instituto de Fermentaciones Industriales (CSIC), Juan de la Cierva, 3, 28006 Madrid, Spain, ${ }^{\star}$ Sección \\ Departamental Ciencias de la Alimentación (Unidad Asociada al CSIC), Universidad Autónoma \\ de Madrid, Campus de Cantoblanco, 28049 Madrid, Spain, ${ }^{\S}$ Bioma Agro Ecology CO, Quartino, \\ Switzerland, and "CNR - Istituto di Scienze dell'Alimentazione (ISA), Avellino, Italy
}

\begin{abstract}
In the present work, an environmentally friendly extraction process using subcritical conditions has been tested to obtain potential natural food ingredients from natural sources such as plants, fruits, spirulina, propolis, and tuber, with the scope of substituting synthetic antioxidants, which are subject to regulation restrictions and might be harmful for human health. A full characterization has been undertaken from the chemical and biochemical point of view to be able to understand their mechanism of action. Thus, an analytical method for profiling the compounds responsible for the antioxidant activity has been used, allowing the simultaneous determination of water-soluble vitamins, fat-soluble vitamins, phenolic compounds, carotenoids, and chlorophylls in a single run. This information has been integrated and analyzed using a chemometrical approach to correlate the bioactive compounds profile with the antioxidant activity and thus to be able to predict antioxidant activities of complex formulations. As a further step, a simplex centroid mixture design has been tested to find the optimal formulation and to calculate the effect of the interaction among individual extracts in the mixture.
\end{abstract}

KEYWORDS: Antioxidant activity; simplex centroid mixture design; polyphenols; vitamins; MLR; PLS; FS; HPLC-DAD profile

\section{INTRODUCTION}

Food additives play an important role in today's complex food supply; nevertheless, they represent one of the most misunderstood topics in food safety raising consumers' concerns. Food control focuses mainly on chemical additives, which although often present are usually only in minor or trace amounts. They are intentionally added to food in order to produce a desired positive effect, although their level has to be maintained within regulated limits. Natural antioxidants are receiving increasing attention in food science because of the reports stating that diets rich in plant antioxidants derived from fruits and vegetables are associated with lower risks of coronary heart disease and cancer $(1,2)$. For example, recent reports have been published suggesting that naturally occurring homologous mixtures have a more beneficial effect than the intake of synthetically produced vitamin E (3). This fact is probably related to the absence of nonactive stereoisomers (4) in synthetic products. Therefore, the importance of finding natural sources of antioxidants is increasing.

Renewed attention in the food industry is being focused on $\mathrm{CO}_{2}$ as a clean technology for ingredients and additives in manufacturing. $\mathrm{CO}_{2}$ extraction has been recognized as one of the new technologies able to extract higher quality natural

*To whom correspondence should be addressed. E-mail: elena@ ifi.csic.es. Fax: +34915 644853 . ingredients, such as food aroma compounds, colorants, antioxidants, and even antimicrobial agents. Several advantages have been demonstrated compared to traditional extraction methods using organic solvents or steam-distillation to extract different compounds from natural sources. For instance, the potential to process natural products at mild temperatures under chemically inert conditions, using $\mathrm{CO}_{2}$ as an extraction fluid, also results in a low environmental impact. Moreover, due to these mild conditions, the functional, sensorial, and nutritional properties of the products are kept unaltered $(5,6)$.

An important challenge in the food industry when dealing with natural additives is to know their exact chemical composition and to be able to correlate it with their biological activity. Therefore, in the present work, a full characterization of the different extracts obtained under subcritical conditions has been undertaken from a chemical and biochemical point of view. The selected method for chemical characterization was based on a previous work carried out in our laboratory (7) for profiling different bioactive compounds such as water-soluble vitamins (ascorbic acid, thiamine, folic, pyridoxine, nicotinamide, cobalamine), fat-soluble vitamins ( $\alpha$-tocopherol, retinol acetate, cholecalciferol), phenolic compounds (phenolic acids, cinnamic acids, flavanones, isoflavones, anthocianins), carotenoids ( $\beta$-carotene and lutein), and chlorophylls in a single run, based on an HPLC-DAD analysis. The complete information obtained using this method can be 
combined with data corresponding to antioxidant capacity (DPPH radical scavenging method) and, once integrated and analyzed using different chemometric approaches, can provide a useful tool to correlate composition and bioactivity, with the added advantage of offering a method to predict antioxidant activities of similar or even more complex formulations.

Experimental designs are highly useful when it comes to optimize several parameters at once or when trying to find the best composition of a mixture of more than two components. A mixture experiment is an special type of experimental design in which the factors are the ingredients or components of a mixture. Among the different mixtures experiments, simplex centroid designs allow one to estimate not only main effects but also significant interactions on a mixture (8).

The present work is focused in the development of novel class of food additives based on substances extracted from natural sources by using environmentally friendly extraction processes, such as near critical carbon dioxide extraction. The use of chemometrics allows one to obtain information about the correlation between chemical composition and bioactivity considering, in a first step, all the compounds involved in the extract, meaning all the compounds giving a characteristic profile of the sample. This is a more comprehensive approach, since the compounds are not discriminated in advance for their supposed (described in the literature), or not, bioactivity. On the contrary, the hypothesis is related to the effectiveness of a profile to provide an specific biological activity. The optimization of the model is carried out by means of multiple linear regression methods whose validity is lately assured by using a simplex centroid design.

\section{MATERIALS AND METHODS}

Subcritical Fluid Extraction. In a first step, dried raw materials (Spirulina pacifica, Citrus compositum, Raphanus niger (radish), Rosmarinus officinalis (rosemary), Propolis, Medicago compositum (alfalfa), Carica papaya (papaya)) were extracted using a hydroethanolic mixture (30:70) and near critical $\mathrm{CO}_{2}$.

Extraction conditions ranged between 68 and $75 \mathrm{~atm}$ and 37 and $45^{\circ} \mathrm{C}$ for each raw material. A total of 10 extraction conditions were evaluated. The equipment used to obtain bioactive extracts was SFT-100 of Supercritical Fluid Technology Inc. (Newark, DE) with adaptations with a $100 \mathrm{~mL}$ extraction vessel. This adaptation has been designed and operated by Bioma Agro Ecology CO (Quartino, Switzerland).

The novelty of this approach is the extraction rationale which is the opposite of the traditional extraction processes. Instead of heating the extraction system in order to shorten the extraction time, the process is carried out near room temperature using a rising pressure on the extraction liquid that interacts with the solid matrix. Extraction at low temperatures is a relevant issue, since it is possible to avoid a thermal stress on thermolabile substances. Various experimental conditions, as described below, were used to determine the conditions that maximized the extraction. The SFE procedures were performed for $60 \mathrm{~min}$ at a flow rate of $5 \mathrm{~mL} / \mathrm{min}$ of liquid $\mathrm{CO}_{2}$.

Chemical Characterization. A HPLC method previously developed in our laboratory (7) was used; under the selected conditions, water-soluble vitamins (ascorbic acid, thiamine, folic, pyridoxine, nicotinamide, cobalamine), fat-soluble vitamins ( $\alpha$-tocopherol, retinol acetate, cholecalciferol), phenolic compounds (phenolic acids, cinnamic acids, flavanones, isoflavones, antocyanins), carotenoids ( $\beta$-carotene and lutein), and chlorophylls (pheophytin, chlorophyll-a and -b) were separated in a single run. Briefly, an ACE- $100 \AA^{\circ} \mathrm{C}_{18}$ column $(150 \mathrm{~mm} \times 4.6 \mathrm{~mm}, 3 \mu \mathrm{m}$ particle size $)$ was used combining isocratic and linear gradient elution with a mobile phase consisting of $0.010 \%$ trifluoroacetic acid (solvent A) and methanol (solvent B) at a flow rate $0.7 \mathrm{~mL} / \mathrm{min}$. The gradient profile (A:B) started at 95:5 and was constant in the first $4 \mathrm{~min}$, then linearly changed up to 2:98 during the next $6 \mathrm{~min}$, then it was constant in the next $7 \mathrm{~min}$, increased up to $0: 100$ in $2 \mathrm{~min}$, then constant until a total analysis time of $40 \mathrm{~min}$, and finally linearly increased up to $95: 5$ to reach initial conditions. The most suitable detection wavelength for simultaneous vitamin-polyphenol determination was $280 \mathrm{~nm}$. Quantification of the different families of compounds was performed by building a calibration curve for each of them, as could be seen in ref 7 .

Functional Characterization. The antioxidant activity was evaluated using two methods: the DPPH radical scavenging test (9) and the total phenolics measurement using Folin reagent (10).

The total phenolics method is based on the original Folin-Ciocalteau method developed in 1927 (11). The measurements were carried out in an absorbance plate-reader, using ELISA 96-well plates of $250 \mu \mathrm{L}$. The method consists of mixing $200 \mu \mathrm{L}$ of $\mathrm{Na}_{2} \mathrm{CO}_{3}(2 \% \mathrm{w} / \mathrm{v}$ in water) with $10 \mu \mathrm{L}$ of extract; after $3 \mathrm{~min}$ of reaction, $5 \mu \mathrm{L}$ of Folin reagent was added. After $30 \mathrm{~min}$ of incubation, the absorbance was measured at $700 \mathrm{~nm}$ (Tecan Sunrise multiwell absorbance reader, Männedorf, Switzerland).

The antioxidant activity of the extracts was measured by the DPPH radical scavenging method. This method is based on that previously developed by Brand-Williams et al. (9) adapted to use 96-microwell plates. The method consisted of measuring the change in absorbance that occurred at $517 \mathrm{~nm}$ by mixing $195 \mu \mathrm{L}$ of $\mathrm{DPPH}^{*}$ solution $(23.5 \mathrm{mg} / \mathrm{L}$ in ethanol) with $5 \mu \mathrm{L}$ of extract. Since reaction time depends on the extract, data were collected every $15 \mathrm{~min}$ for $5 \mathrm{~h}$. The stabilization time occurred when a plateau was reached, the end of the reaction for each sample.

Statistical Analysis. In order to find the main components of the extracts that contribute to its antioxidant activity, data related to the measured antioxidant activity and vitamin-phenolic HPLC profiles were submitted to multiple linear regression (MLR) analysis using forward stepwise (fs) and partial least squares (PLS). An equation of the form $\hat{y}=$ $b_{0}+\sum_{i=1}^{p} b_{i} X_{i}$ is assumed, where $b_{0}$ is the intercept of the model, $b_{i}$ is the regression coefficient for the $i$ th compound $\left(X_{i}\right), p$ is the number of compounds in the model, and $\hat{y}_{i}$ is the antioxidant activity calculated using the model. All calculations were done with the STATISTICA software for Windows, version 7.1 (StatSoft, Tulsa, OK, 2006, http://www.statsoft. com), using the forward stepwise procedure in the Statistics, Multiple Regression module (with values of 4.0 and 3.9 for F-to-enter and F-toremove, respectively, and fixing a limit of 10 steps) and the partial least squares regression procedure in the Statistics, Advanced Linear/Nonlinear Model module of STATISTICA program.

Mixture Experimental Design. The design of the antioxidant additive and its composition was studied by means of a modified simplex centroid design (12). The components of the mixture were selected among those with higher contribution to the antioxidant activity. This kind of modified simplex centroid with face experiments design is a mixture design for the full quadratic model with more runs than the classic design.

\section{RESULTS AND DISCUSSION}

Extraction conditions ranged between 68 and $75 \mathrm{~atm}$ and 37 and $45^{\circ} \mathrm{C}$. As can be seen, this is a relatively small area of experimental conditions; in fact, these ranges could be a normal variation in an industrial scale extraction plant. Our aim was to cover this area to appreciate the effect of normal variation, where pressure and temperature cannot be controlled as easily as on a laboratory scale. By using these extraction conditions, $\mathrm{CO}_{2}$ expands its liquid phase (water/ethanolic) and reduces its viscosity (13), increasing the mass transfer rate.

When the antioxidant activity of vegetable extracts was measured, three levels of activity were found in the DPPH radical scavenging test: low (citrus, propolis, and raphanus), medium (spirulina, alfalfa, and papaya), and high (rosemary) antioxidant. On the other hand, the activity of the extracts showed the same trend when plotting antioxidant activity $\left(\mathrm{EC}_{50}\right)$ versus total polyphenolic content, as can be seen in Figure $\mathbf{1}$.

Chemical characterization was done using a method previously developed in our laboratory (7). This screening method allows one to quantify simultaneously several kinds of bioactive compounds, namely, vitamins (hydro- and fat-soluble), phenolic compounds, and certain pigments such as chlorophylls and carotenoids. This method proved its efficacy in juices, fortified juices, beers, and milky drinks (7). 


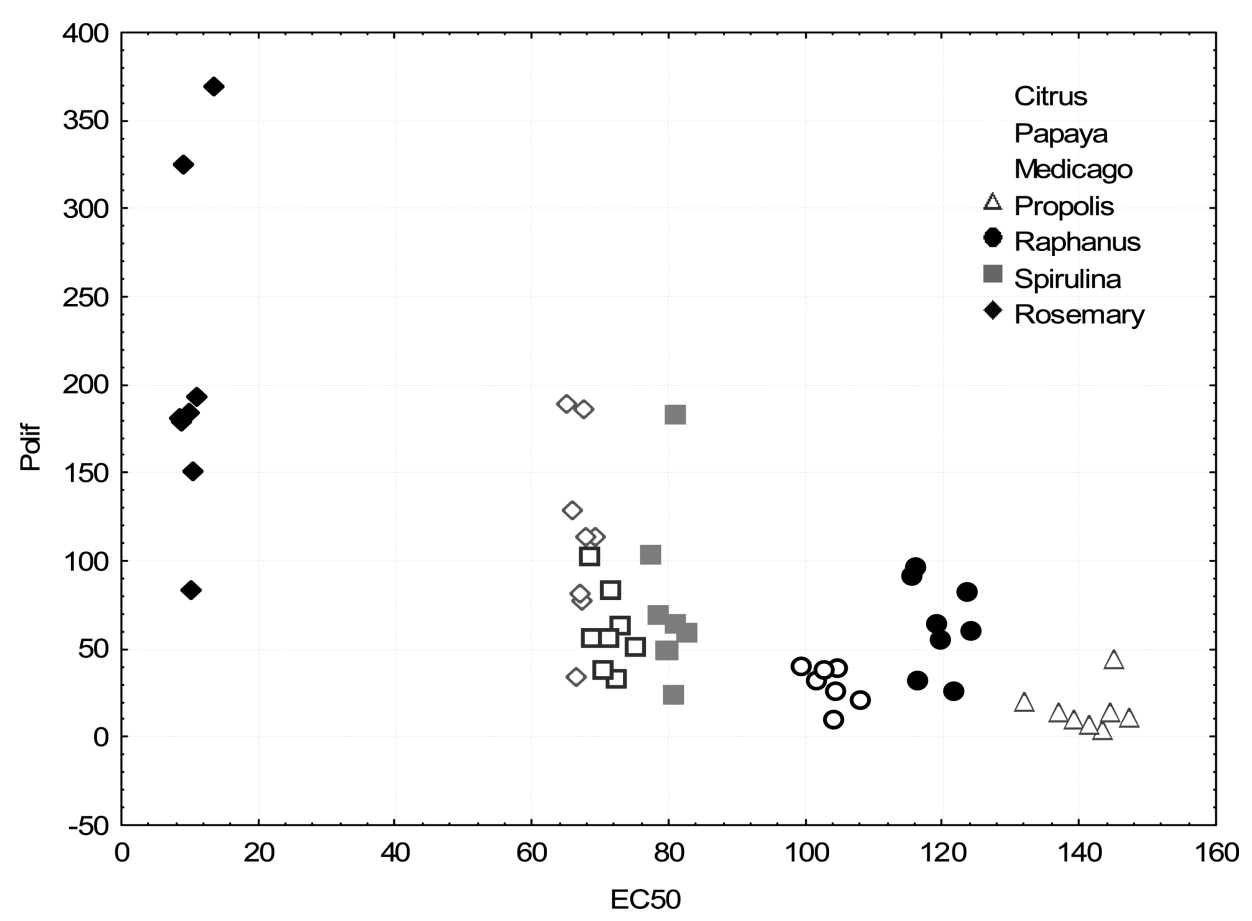

Figure 1. Radical scavenging activity $\left(\mathrm{EC}_{50} \mathrm{mg} / \mathrm{g}\right.$ ) versus total polyphenolic content ( $\mathrm{mg}$ gallic acid equiv/g extract).

In general terms, three zones were easily differentiated in the chromatograms: water-soluble vitamins $(2-12 \mathrm{~min})$, phenolic compounds (10-20 min), and, finally, fat-soluble vitamins and pigments $(20-40 \mathrm{~min})$. Representative chromatograms of the different raw materials are shown in Figure 2. Table 1 shows the mean composition of the different raw materials considering the different families of compounds detected in the samples. As a matter of fact, individual components were identified tentatively and classified among the different groups or families of compounds, such as phenolic acids, cinnamic acids, flavanones, isoflavones, and antocyanins and numbered according to their retention time; in order to simplify the table, the quantification is presented as the total content of the different groups of compounds, but the statistical analysis was applied considering the individual contribution of each compound corresponding to the different families. The quantification of individual compounds of each family was performed.

A first analysis of the data obtained showed the absence of fatsoluble vitamins. Only a small amount of $\alpha$-tocopherol could be detected in some rosemary extract, but below the quantification level. The main vitamin found in the extracts was thiamine followed by nicotinamide and ascorbic acid. The HPLC analysis of the different extracts revealed, as expected, the presence of rosmarinic acid and carnosic acid and their derivatives in rosemary extracts, providing a high antioxidant activity. Through the study of the chromatographic profiles of the different extracts, it can be inferred that the medium antioxidant activity of Spirulina, alfalfa (Medicago composita) and papaya can be attributed to the presence of carotenoids and phenolic compounds and their synergistic effects. Also, many flavonoids have been detected in extracts from propolis, alfalfa, rosemary, raphanus, and fruits, and most of them have been related to the antioxidant and antimicrobial activity of the $\mathrm{CO}_{2}$-alcoholic and near-critical extracts (14-17).

Statistical Analysis. In order to identify the main components of the extracts that contribute to their antioxidant activity, statistical analysis was performed considering antioxidant activities and the vitamin-phenolic HPLC profiles allowing for the individual contribution of each compound in the whole profile. Two different statistical methods were used: FSMLR (forward stepwise multiple linear regression) and PLS (partial least squares).

When MLR using the FS procedure was applied to find the main components of the extracts that contribute to their antioxidant activity, the selected variables in the vitamin-phenolic HPLC profile were Flavonol15, Flavanone18, Cinnamic acid21, Flavanone15, Flavonol19, Cinnamic acid28, Flavanone13, Flavanone14, Flavonol23, and Flavonol13. These compounds have been numbered according to their retention time, as mentioned above. Results of the regression provide the following equation:

$$
\begin{aligned}
\text { antioxidant activity }= & 77.80+227.25 \\
& \times(\text { Cinnamic } 21)+3.17 \\
& \times(\text { Flavanone13 })+11.04 \\
& \times(\text { Flavanone14 })-7.77 \\
& \times(\text { Flavonol13 })+42.96 \\
& \times(\text { Cinnamic } 28)+5.71 \\
& \times(\text { Flavanone15 })-4.71 \\
& \times(\text { Flavonol15 })+7.46 \\
& \times(\text { Flavonol19 })+0.96 \\
& \times(\text { Flavanone } 18)+6.05 \\
& \times(\text { Flavonol23 })
\end{aligned}
$$

The value of determination coefficient was $R^{2}=0.917$, and the value of the root-mean-square error of calibration (RMSEC) was 10.59. RMSEC is defined by the equation: $\mathrm{RMSEC}=\left[\sum\left(y_{i}-\right.\right.$ $\left.\left.\hat{y}_{i}\right)^{2} / n\right]^{1 / 2}$, where $n$ is the number of samples (in this study $n=60$ ), $y_{i}$ is the observed antioxidant activity, and $\hat{y}_{i}$ is the antioxidant activity calculated using eq $\mathbf{1}$. Figure $\mathbf{3 A}$ shows the calculated values versus the observed values for antioxidant activity. As can be seen, the fit for the predictions of antioxidant activity can be considered appropriate. Despite the proven antioxidant activity of some identified compounds such as ascorbic acid (vitamin C) (18), these compounds are not indicated by the FS-MLR test as being 10 main contributors to the antioxidant activity; neither to increase $\mathrm{EC}_{50}$ (positive coefficient) nor to decrease it 


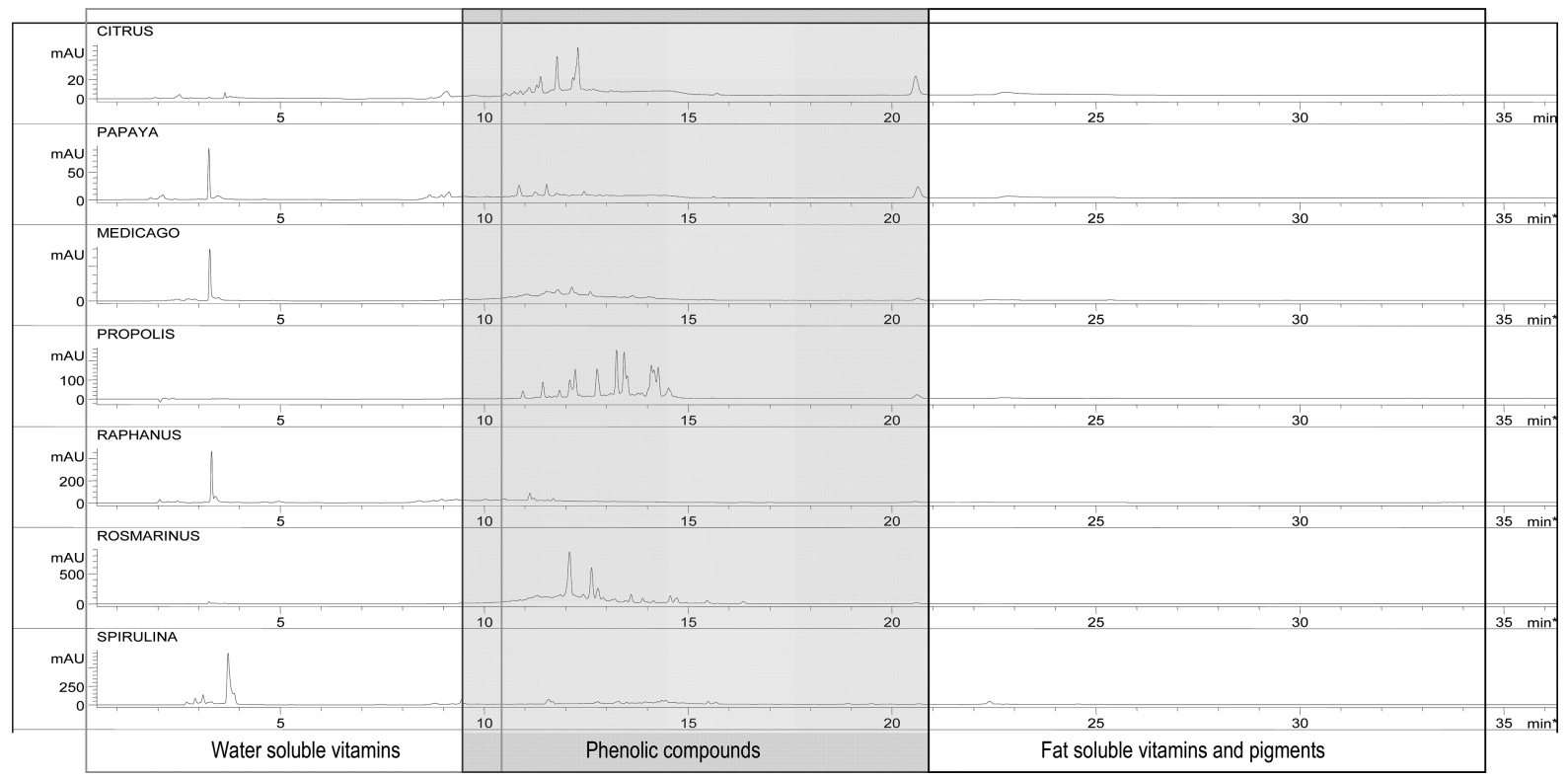

Figure 2. HPLC-DAD chromatograms showing the different regions of bioactive compounds.

Table 1. Mean Composition and Standard Deviation of the Different Extracts of Raw Materials

\begin{tabular}{|c|c|c|c|c|c|c|c|}
\hline & Citrus & Papaya & Alfalfa & Propolis & Raphanus & Rosmarinus & Spirulina \\
\hline cinnamic acids (mg/g) & $0.62 \pm 0.06$ & $0.29 \pm 0.04$ & & $63.78 \pm 17.81$ & $1.19 \pm 0.11$ & & \\
\hline flavanones $(\mathrm{mg} / \mathrm{g})$ & $10.49 \pm 0.88$ & & & $42.34 \pm 6.53$ & & $69.06 \pm 7.90$ & \\
\hline flavonols $(\mathrm{mg} / \mathrm{g})$ & $1.39 \pm 0.19$ & & $21.22 \pm 2.35$ & $9.82 \pm 1.78$ & & $100.50 \pm 10.03$ & \\
\hline phenolic acids (mg/g) & & $0.76 \pm 0.13$ & & & & $19.00 \pm 2.57$ & \\
\hline anthocyan (mg/g) & & $1.89 \pm 0.21$ & & & & & \\
\hline isoflavones (mg/g) & & & $6.15 \pm 0.79$ & & & & \\
\hline rosmarinic acid $(\mathrm{mg} / \mathrm{g})$ & & & & & & $22.41 \pm 2.68$ & \\
\hline carotenoids $(\mathrm{mg} / \mathrm{g})$ & & & $0.72 \pm 0.11$ & & & & $1.00 \pm 0.14$ \\
\hline chlorophylls (mg/g) & & & $1.35 \pm 0.18$ & & & & $2.16 \pm 0.33$ \\
\hline ascorbic acid (mg/g) & $0.52 \pm 0.10$ & $8.66 \pm 1.28$ & $19.34 \pm 3.80$ & & $4.48 \pm 0.82$ & & $26.98 \pm 2.55$ \\
\hline thiamine $(\mathrm{mg} / \mathrm{g})$ & $9.07 \pm 1.33$ & $38.62 \pm 4.86$ & $172.28 \pm 13.14$ & $6.19 \pm 1.89$ & $80.04 \pm 4.86$ & $19.18 \pm 2.78$ & $258.54 \pm 9.91$ \\
\hline nicotinamide $(\mathrm{mg} / \mathrm{g})$ & $5.83 \pm 0.79$ & $8.50 \pm 2.14$ & & & $9.10 \pm 0.50$ & & \\
\hline total phenolics ${ }^{a}$ & $26.45 \pm 2.54$ & $69.54 \pm 9.29$ & $101.93 \pm 9.37$ & $13.97 \pm 4.84$ & $185.27 \pm 8.38$ & $54.36 \pm 20.46$ & $69.82 \pm 9.44$ \\
\hline antioxidant activity $^{b}$ & $103.57 \pm 0.56$ & $71.49 \pm 0.52$ & $67.70 \pm 4.29$ & $141.55 \pm 1.42$ & $119.61 \pm 6.89$ & $10.47 \pm 4.49$ & $80.80 \pm 3.52$ \\
\hline
\end{tabular}

${ }^{a}$ Total phenolics expressed as mg gallic acid equiv/g. ${ }^{b}$ Antioxidant activity expressed as $\mathrm{EC}_{50} \mu \mathrm{g} / \mathrm{mL}$.
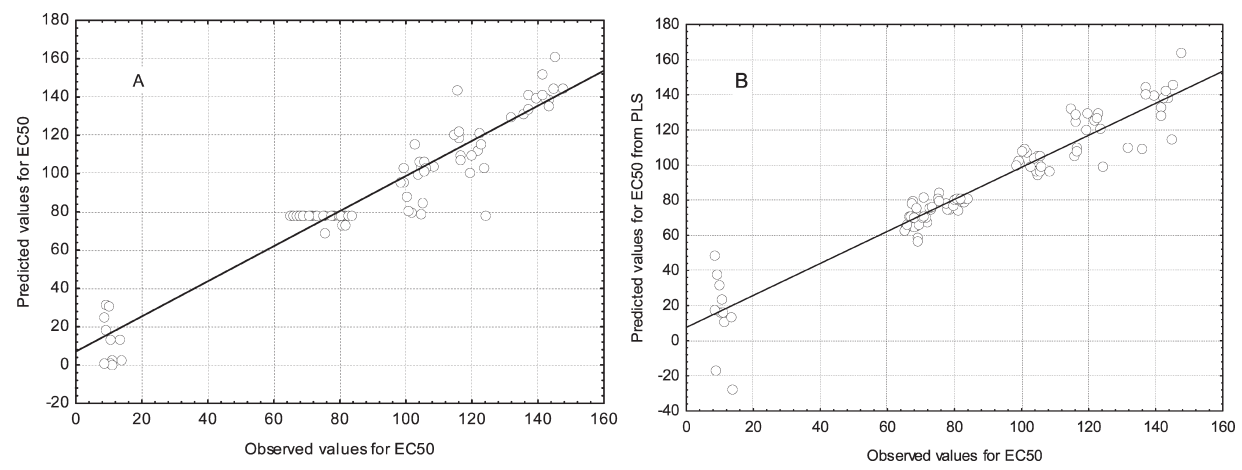

Figure 3. Scatter plot of predicted values for $\mathrm{EC}_{50}$ from MLR using FS (A) and PLS (B) procedures versus observed values.

(negative coefficient). The same procedure was followed but grouping the compounds in families, but the adjustment obtained was very poor $\left(R^{2}<0.7\right)$.

On the other hand, when MLR using the PLS procedure was applied to the prediction of antioxidant activity from all the compounds involved in the complete HPLC profile, three main components were selected by cross-validation, and values of $R^{2}=$ 0.857 and RMSEC $=16$ were obtained. Figure 3B shows the predicted values for antioxidant activity from MLR using the PLS procedure versus the observed values. As can be seen, the fit can be considered as also appropriate.

Mixture Design. Although it is highly useful to know how the chemical composition (or profile) affects the antioxidant activity of a specific extract, it is almost impossible to adjust each compound individually because extracts come from natural matrices, whose compositions are extremely complex. Mixture 


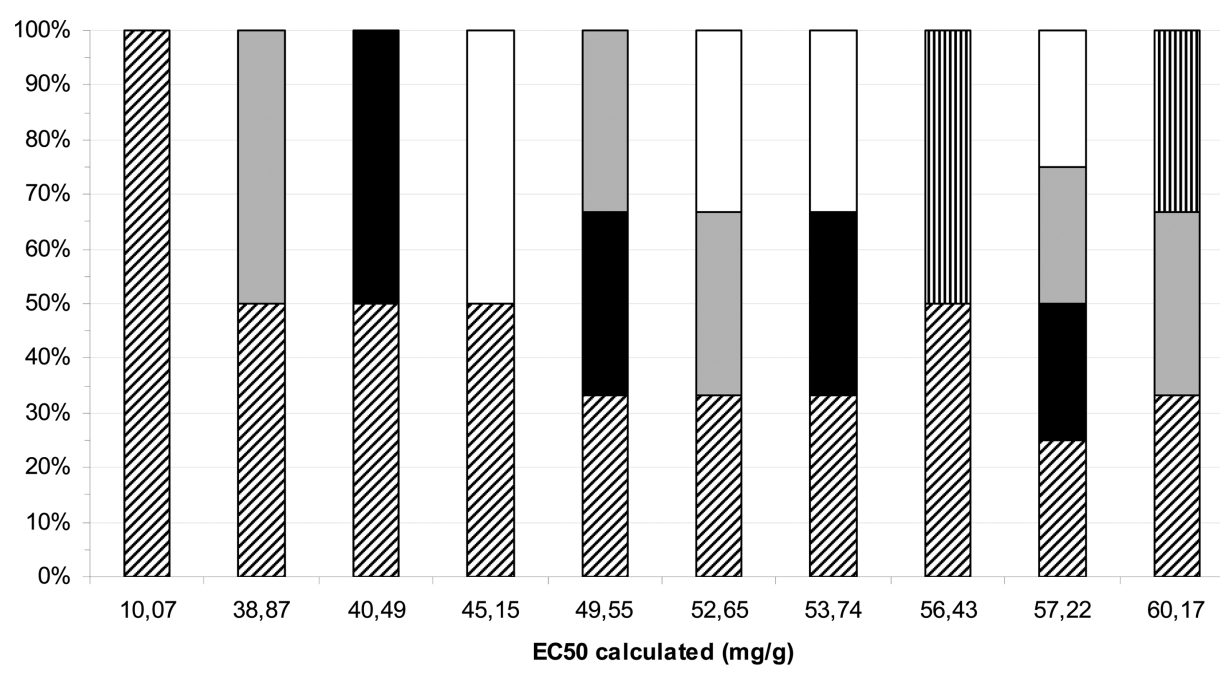

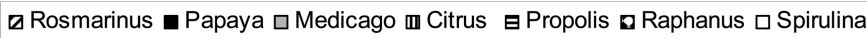

Figure 4. Prediction of antioxidant activity of mixtures by using the 7 factor simplex lattice design; best 10 antioxidant mixtures are shown.

experiments are conducted to determine if mixed blends (or formulations) are more desirable than additives obtained using a single raw material. An important property of the mixture experiment is that the change in the response depends on the proportionality of the individual components present in the mixture and not on the amount of the mixture (8).

The analysis of variance of the extraction conditions (considering the different experiments) versus antioxidant activity only showed statistically significant differences among different batches of Spirulina and alfalfa. On the other hand, although such differences can be found in some values of antioxidant activity of some extracts, the real values of antioxidant activity are close enough $\left( \pm 7 \mu \mathrm{g} / \mathrm{mL}\right.$ of the difference in $\mathrm{EC}_{50}$ values around $70-80 \mu \mathrm{g} / \mathrm{mL}$ ) to consider that extraction conditions do not strongly affect the results obtained in terms of antioxidant activity. Therefore, by using this approach, each product (raw material) was considered independent of the extraction conditions (in the assayed range), and thus, 10 values were considered and a mean and standard deviation were obtained. To be able to design a formulation considering the different raw materials, a PLS methodology was used, and eq $\mathbf{2}$ was obtained, as follows:

$$
\begin{aligned}
\text { antioxidant activity }= & 102.78 \times \text { Citrus }+70.91 \\
& \times \text { Papaya }+67.67 \\
& \times \text { Alfalfa }+140.54 \\
& \times \text { Propolis }+118.81 \\
& \times \text { Raphanus }+10.07 \\
& \times \text { Rosmarinus }+80.23 \times \text { Spirulina }
\end{aligned}
$$

In this equation, a linear model of composition ( 7 factor simplex lattice) was considered, and therefore, no synergies were taken into account; each term is composed by the relative amount of the raw material (expressed in \%) and a coefficient that gives an estimation of the relative contribution of each raw material to the total antioxidant activity (expressed as $\mathrm{EC}_{50}$ ). As can be seen in Figure 4, the best results (lowest $\mathrm{EC}_{50}$ values) are those with high proportions of rosemary $(100 \%$ and $50 \%)$, followed by medicago $(50 \%)$ and papaya $(50 \%)$. The main disadvantage of using this model is the lack of prediction in case of synergies or inhibitions among different compounds coming from different raw materials.

In order to demonstrate the ability of our approach to predict the antioxidant activity of a mixture of different raw materials, a

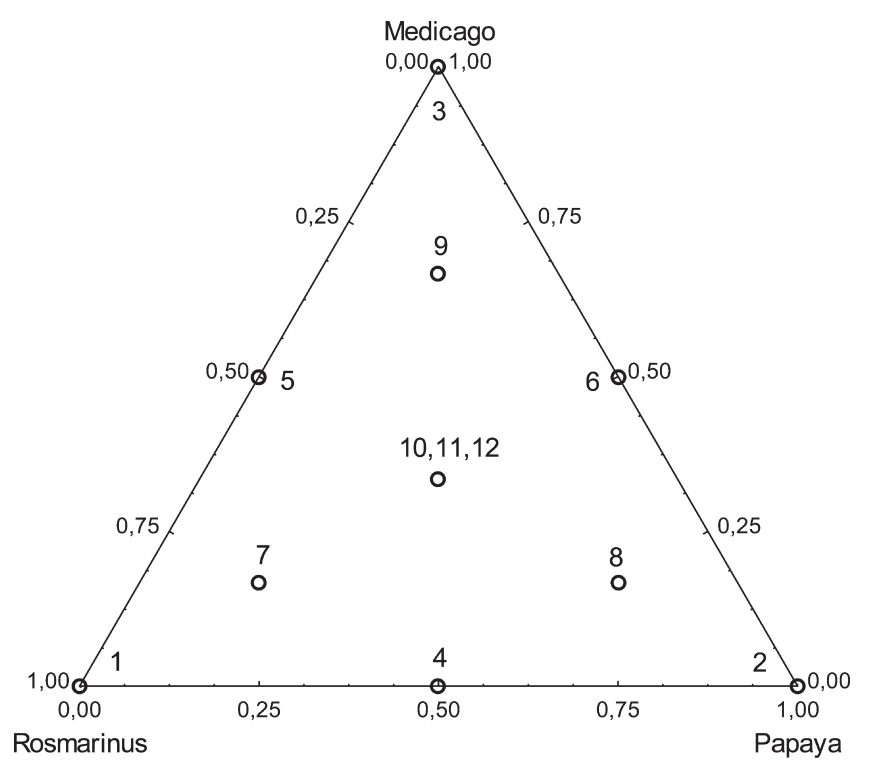

Figure 5. Ternary diagram illustrating the level combinations of rosemary, papaya, and alfalfa in the modified simplex centroid design.

systematic study was performed using a mixture experiment. The final goal was, with this model, to be able to find the optimum formulation, that is, the one providing the best antioxidant activity/profile. Therefore, a modified simplex centroid design for mixtures, with three components at different concentrations (prepared on a dry basis), was performed; the graphical planning of the design can be seen in Figure 5, and the levels of the factors in Table 2. Experiments 1, 2, and 3 were vertex points of the design corresponding to pure components. Points 4 , 5, and 6 on the sides were 1:1 binary mixtures, while points 7,9 , and 9 corresponded to mixtures of 1:1:4, 1:4:1, and 4:1:1 proportions, respectively. Points 10,11 , and 12 were ternary mixtures with equal parts of all three components. The design was run by using three more antioxidant materials, namely, rosemary, alfalfa, and papaya. All the mixtures were prepared in percentages of dry weight and redissolved in ethanol/water (1:1). The batch selected to run the design was batch 2 of each raw material, and the extraction conditions used were $71.5 \mathrm{~atm}$ and $41{ }^{\circ} \mathrm{C}$. A linear predictive model including interactions ( $n=12$ runs) was considered. 
Table 2. Comparison of Antioxidant Activity of Formulations Obtained with Modified Simplex Centroid Design for Mixtures

\begin{tabular}{llllcc}
\hline$\#$ & Rosmarinus & Papaya & Alfalfa & $E_{50}$ (observed) $^{a}$ & $E_{50}$ \\
\hline 1 & 1 & 0 & 0 & 9.11 & 10.07 \\
2 & 0 & 1 & 0 & 70.61 & 70.91 \\
3 & 0 & 0 & 1 & 65.32 & 67.67 \\
4 & 0.5 & 0.5 & 0 & 44.07 & 40.49 \\
5 & 0.5 & 0 & 0.5 & 37.25 & 38.87 \\
6 & 0 & 0.5 & 0.5 & 69.57 & 69.29 \\
7 & 0.6667 & 0.1667 & 0.1667 & 28.50 & 29.81 \\
8 & 0.1667 & 0.6667 & 0.1667 & 63.26 & 60.23 \\
9 & 0.1667 & 0.1667 & 0.6667 & 54.81 & 58.61 \\
10 & 0.3333 & 0.3333 & 0.3333 & 46.88 & 49.55 \\
11 & 0.3333 & 0.3333 & 0.3333 & 46.11 & 49.55 \\
12 & 0.3333 & 0.3333 & 0.3333 & 48.85 & 49.55 \\
\hline
\end{tabular}

${ }^{a}$ Antioxidant activity expressed in $\mathrm{EC}_{50}(\mu \mathrm{g} / \mathrm{mL})$

Results of the measured antioxidant activity obtained in the mixture design model can be seen in Table 2 (observed values of $\mathrm{EC}_{50}$ ). In fact, the statistical analysis of the data of simplex centroid design gives eq $\mathbf{3}$ with a determination coefficient equal to $R^{2}=0.993$.

$$
\begin{aligned}
\text { antioxidant activity }= & 9.33 \times \text { Rosmarinus }+71.78 \\
& \times \text { Papaya }+65.29 \times \text { Alfalfa }+9.51 \\
& \times \text { Rosmarinus } \times \text { Papaya }-9.59 \\
& \times \text { Rosmarinus } \times \text { Alfalfa }-1.41 \\
& \times \text { Papaya } \times \text { Alfalfa }
\end{aligned}
$$

This equation correlates antioxidant activity with the composition of the formulation. The calculated values for $\mathrm{EC}_{50}$ with this equation are shown in Table 2. The differences among the observed and calculated values of the 12 formulations are close enough to confirm the validity of the fitted eq $\mathbf{2}$ and the validity of the suggested approach. Since the $P$-value of the F-test is greater than or equal to 0.05 , there is not a statistically significant difference between calculated and observed values at the $95.0 \%$ confidence level, as stated by the analysis of variance performed on Statgraphics Plus 5.1. As can be seen, certain synergy can be appreciated among rosemary and alfalfa and among papaya and medicago, since these terms have negative coefficients (the lower the $\mathrm{EC}_{50}$, the higher the antioxidant activity). On the other hand, the positive interaction coefficient among rosemary and papaya indicates that their mixtures would present higher values of $\mathrm{EC}_{50}$, which means lower antioxidant activity.

From these results, it can be seen that pure rosemary presents the higher antioxidant activity, although if other raw materials could be used because of the flavor, the price, or the need of other compounds in the mixture (such as carotenoids and vitamins), it is possible to predict, with the methodology shown in the present work, the antioxidant activity of the final formulation in a simple way.

In conclusion, the results presented in this work showed the possibility to design an antioxidant ingredient produced using different vegetable extracts, obtained using near-critical fluids, as food preservative agents for the food industry. By using a chemometric approach, the design of the antioxidant ingredient 376 (additive-formulation) requires minimum experimental effort, 377 which is ensured by a well-planned experimental design.

\section{LITERATURE CITED}

(1) Harris, A.; Devaraj, S.; Jialal, I. Oxidative stress, alpha-tocopherol therapy, and atherosclerosis. Curr. Atheroscler. Rep. 2002, 4, 373-380.

(2) Brigelius-Flohe, R.; Kelly, F. J.; Salonen, J. T.; Neuzil, J.; Zingg, J.-M.; Azzi, A. The European perspective on vitamin E: current knowledge and future research. Am. J. Clin. Nutr. 2002, 76, 703-716.

(3) National Institute for Health. Facts about Dietary SuplementsVitamin E; Warren Grant Magnuson Clinical Center: MD, 2002.

(4) Brigelius-Flohe, R.; Traber, M. G. Vitamin E: function and metabolism. FASEB J. 1999, 13, 1145-1155.

(5) King, M. B.; Bott, T. R. Extraction of natural products using nearcritical solvents; Blackie Academic \& Professional: Glasgow, 1993.

(6) Mukhopadhyay, M. Natural extracts using supercritical carbon dioxide; CRC Press: Boca Raton, FL, 2000; p 339.

(7) Mendiola, J. A.; Marin, F. R.; Señoráns, F. J.; Reglero, G.; Martín, P. J.; Cifuentes, A.; Ibáñez, E. Profiling of different bioactive compounds in functional drinks by HPLC. J. Chromatogr., A 2008, 1188, 234-241.

(8) Bondari, K. In Mixture Experiments and Their Applications in Agricultural Research; SAS Users Group International: Philadelphia, 2005; pp 209-230.

(9) Brand-Williams, W.; Cuvelier, M. E.; Berset, C. Use of a free radical method to evaluate antioxidant activity. Lebensm. Wiss. Technol. 1995, 28, 25-30.

(10) Cuvelier, M. E.; Richard, H.; Berset, C. Antioxidative activity and phenolic composition of pilot-plant and commercial extracts of sage and rosemary. J. Am. Oil Chem. Soc. 1996, 73, 645-652.

(11) Folin, O.; Ciocalteu, V. On tyrosine and tryptophane determinations in proteins. J. Biol. Chem. 1927, 73, 627-650.

(12) Gustavsson, A.; Sundkvist, J. E. Design and optimization of modified simplex methods. Anal. Chim. Acta 1985, 167, 1-10.

(13) Sih, R.; Armenti, M.; Mammucari, R.; Dehghani, F.; Foster, N. R. Viscosity measurements on saturated gas-expanded liquid systemsEthanol and carbon dioxide. J. Supercrit. Fluids 2008, 43, 460-468.

(14) Scazzocchio, F.; D’Auria, F. D.; Alessandrini, D.; Pantanella, F. Multifactorial aspects of antimicrobial activity of propolis. Microbiol. Res. 2006, 161, 327-333.

(15) Tripoli, E.; Guardia, M. L.; Giammanco, S.; Majo, D. D.; Giammanco, M. Citrus flavonoids: Molecular structure, biological activity and nutritional properties: A review. Food Chem. 2007, 104, 466-479.

(16) Friedman, M. Overview of antibacterial, antitoxin, antiviral, and antifungal activities of tea flavonoids and teas. Mol. Nutr. Food Res. 2007, 51, 116-134.

(17) Lule, S. U.; Xia, W. Food phenolics, pros and cons: A review. Food Rev. Int. 2005, 21, 367-388.

(18) Halliwell, B. Antioxidants in human health and disease. Annu. Rev. Nutr. 1996, 16, 33-50.

Received for review June 8, 2009. Revised manuscript received December 2, 2009. Accepted December 2, 2009. This work has been funded by the NOCHEMFOOD project (STREP FP6-23060-2006, European Commission, Sixth Framework Program of Research and Development, Food Quality and Safety Thematic Priority).

\section{0}

381

382

383

384

385

386

387

388

389

390

391

392

393

394

395

396

397

398

399

400

401

402

403

404

405

406

407

408

409

410

411

412

413

414

415 